\title{
ANL/MSD/PP- -84691
}

DFC $\cap 61996$
Direct Evidence for, and the Nature of, Josephson Coupling between Cu-O Bilayers in a Highly-Anisotropic Superconductor*

\author{
J.D. Hettinger, B.W. Veal, Jr., A.P. Paulikas, P. Kostic, K.E. Gray, B.R. Washburn, \\ W.C. Tonjes, and A.C. Flewelling \\ Materials Science Division \\ Argonne National Laboratory, Argonne, Illinois 60439
} The submitted manuscript has been authored
by a contractor of the U.S. Government under
contract No. W-31-109-ENG-38. Accordingly.
the U.S. Government retains a nonexdusive,
royally-free license to publish or reproduce the
published torm of this contribution, or allow
others to do so, for U.S. Government purposes.

\section{DISCLAIMER}

This report was prepared as an account of work sponsored by an agency of the United States Government. Neither the United States Government nor any agency thereof, nor any of their employees, makes any warranty, express or implied, or assumes any legal liability or responsibility for the accuracy, completeness, or usefulness of any information, apparatus, product, or process disclosed, or represents that its use would not infringe privately owned rights. Reference herein to any specific commercial product, process, or service by trade name, trademark, manufacturer, or otherwise does not necessarily constitute or imply its endorsement, recommendation, or favoring by the United States Government or any agency thereof. The views and opinions of authors expressed herein do not necessarily state or reflect those of the United States Government or any agency thereof.

\footnotetext{
${ }^{*}$ Work partially supported by the U.S. Department of Energy, Division of Basic Energy Sciences-Materials Sciences, and Energy Efficiency and Renewable Energy, as part of a program to develop electric power technology, under contract \#W-31-109-ENG-38, by the National Science Foundation Office of Science and Technology Centers under contract DMR \#91-20000, and by the Division of Educational Programs at ANL.
} 


\section{DISCLAIMER}

Portions of this document may be illegible in electronic image products. Images are produced from the best available original document. 
Direct Evidence for, and the Nature of, Josephson Coupling between $\mathrm{Cu}-\mathrm{O}$ Bilayers in a Highly-Anisotropic Superconductor

\author{
J.D. Hettinger, B.W. Veal, Jr., A.P. Paulikas, P. Kostic, K.E. Gray, B.R. Washburn, \\ W.C. Tonjes and A.C. Flewelling \\ Materī̄lls Sciences Division, Argonne National Laboratory \\ Argonne, Illinois, 60439
}

The submitted manuscript has
been authored by a contractor of the .
U.S. Government under contract No.
W-31-109-ENG-38. Accordingly, the.
U.S. Government retains a
nonexclusive, royalty-free license to
publish or reproduce the published
form of this contribution, or allow
others to do so, for U.S. Government
purposes.

PACS \# 74.40+k, 74.60.Ge, 74.50.+r

\begin{abstract}
The angular-dependent, $\mathrm{c}$-axis resistivity for oxygen-deficient $\mathrm{YBa}_{2} \mathrm{Cu}_{3} \mathrm{O}_{7-\bar{\delta}}$ single crystals is shown to be a maximum for fields parallel to the c-axis, i.e., for zero macroscopic Lorentz force, and agrees with a series stack of Josephson tunnel. junctions. The c-axis component of field dominates the c-axis dissipation in most cases. The results indicate the possibility of an unusual normal-state c-axis conductance and that the c-axis junctions may be extremely underdamped. [word count $=80$ ]
\end{abstract}


Recent experiments on ${ }^{1,2} \mathrm{Bi}_{2} \mathrm{Sr}_{2} \mathrm{CaCu}_{2} \mathrm{O}_{8}$ and ${ }^{2} \mathrm{Tl}_{2} \mathrm{Ba}_{2} \mathrm{Ca}_{2} \mathrm{Cu}_{3} \mathrm{O}_{10}$ crystals related to the c-axis conduction in highly-anisotropic, high-temperature superconductors (HTS) and a subsequent interpretation ${ }^{3}$ of the field dependence found in Ref. 1 have strongly suggested that these HTS materials behave as a stack of Josephson junctions between close, well-coupled, bi- or tri-layers of $\mathrm{Cu}-\mathrm{O}$. The strong, 1/B dependence of Josephson coupling energy, $\mathrm{E}_{j}$, on the field, B, parallel to the c-axis, used in Ref. 3 , was first proposed 4 to understand the irreversibility behavior of the ab-plane resistivity for fields parallel to the c-axis. While such a field dependence was in quantitative agreement with measurements ${ }^{5}$ in discrete, thin-film $\mathrm{Nb}$-based junctions, a more convincing test of this aspect of the model for HTS requires direct c-axis resistivity measurements. This also eliminates the uncertain absolute magnitude of $\mathrm{E}_{\mathrm{j}}$ inherent in the analysis of Ref. 4 .

Thus we report measurements of the c-axis resistance, as a function of magnetic field and its orientation with respect to the c-axis, using oxygen-deficient, and thus reasonably anisotropic $6, \mathrm{YBa}_{2} \mathrm{Cu}_{3} \mathrm{O}_{7-\delta}$ single crystals $\left(\mathrm{T}_{\mathrm{c}}=35-75 \mathrm{~K}\right)$. The measured angular-dependent c-axis resistance, at temperatures somewhat below the transition temperature, $T_{c}$, exhibits a maximum in dissipation for fields parallel to the c-axis, i.e., for zero macroscopic Lorentz force: this tends to rule out ${ }^{7}$ the motion of vortices from the external field. The dependences of the dissipation on applied field strength and angle with respect to the c-axis are in excellent agreement with the field dependence predicted by Refs. 3-5 for a series stack of Josephson tunnel junctions, assuming that only the c-axis component of the field plays a role ${ }^{8}$. The data define a Josephson coupling energy 9,10 which shows the same field dependence as found ${ }^{5}$ in the discrete, thin-film $\mathrm{Nb}$-based junctions.

Single crystals, grown using a self-flux method 11 , had their oxygen composition modified (reduced) by annealing for 200 hours at $500^{\circ} \mathrm{C}$ in appropriate mixtures ${ }^{12}$ of. $\mathrm{O}_{2}$ in $\mathrm{N}_{2}$ such that $0.095,3.7$ and $8.3 \% \mathrm{O}_{2} / \mathrm{N}_{2}$ yielded $\mathrm{T}_{\mathrm{c}}=35 ; 65$ and $75 \mathrm{~K}$ as measured. by electrical transport. Silver epoxy was placed on the plate-like crystal surfaces $(\sim 1 \mathrm{~mm} \times 1 \mathrm{~mm} \times 50 \mu \mathrm{m})$ before annealing. The Ag diffuses into the crystal during the oxygen anneal to form an electrical contact pad to which Au leads were attached, two on each face. Large current pads decrease heating effects and improve current uniformity. For very large resistivity anisotropies, currents redistribute rapidly in the ab-planes creating an effectively uniform c-axis current. This uniformity was confirmed by exchanging current and voltage leads on the bottom (only) of the crystal and getting identical results. More isotropic samples than used here (i.e., higher $T_{C}$ ) failed this test because of the (relatively) smaller c-axis resistivity. 
Measurements of dc electrical transport properties were carried out in a $7 \mathrm{~T}$ splitcoil radial-access magnet in which the sample probe is rigidly mounted on a rotational feedthrough which was stepper-motor controlled with a reproducibility of better that 0.01 degrees. Voltage-current measurements confirmed that the data were always collected in the linear response regime (using $\mathrm{J} \sim 1 \mathrm{~A} / \mathrm{cm}^{2}$ ).

The c-axis resistance, $\mathrm{R}_{c}$, of an oxygen-deficient $\mathrm{YBa}_{2} \mathrm{Cu}_{3} \mathrm{O}_{7-\delta}$ single crystal with $T_{C} \sim 65 \mathrm{~K}$ is shown in Fig. 1 as a function of the angle, $\phi$, between the field direction and the $\mathrm{c}$-axis for a temperature of $60 \mathrm{~K}$ and a field, $\mathrm{B}$, of $1 \mathrm{~T}$. The maximum $\mathrm{R}_{\mathrm{c}}$ - occurs for Bllc, which corresponds to a minimum macroscopic Lorentz force on the Abrikosov vortices and it drops over three orders-of-magnitude into the noise for the maximum Lorentz force, i.e., Bllab. This tends to rule out ${ }^{7}$ the motion of vortices from the external field as the cause of the dissipation, but it is shown below to be consistent with dissipation across inter-bilayer Josephson junctions.

At reduced temperatures, $\mathrm{t}=\mathrm{T} / \mathrm{T}_{\mathrm{c}}$, closer to one, dissipation due to flux motion ${ }^{13}$. can be relatively more important because the pinning energy is $\sim(1-t)^{2}$, while the Josephson coupling is (1-t). Such additional dissipation is clearly seen nearer to $T_{C}$ but only for fields close to the minimum-pinning, maximum-Lorentz-force, abplane direction. We do not discuss this result further, but it emphasizes the clear distinction between dissipation across Josephson junctions and that due to motion of vortices from the external field.

We also measured $R_{c}(T)$ at $\phi=0$ as a function of $B$ and determined the activation energy, $U$, from Arrhenius plots. These results are shown in Fig. 2 for two $\mathrm{YBa}_{2} \mathrm{Cu}_{3} \mathrm{O}_{7-\delta}$ crystals $\left(\mathrm{T}_{\mathrm{c}}=65\right.$ and $\left.75 \mathrm{~K}\right)$, together with identical determinations.made on two discrete, thin-film $\mathrm{Nb}$ junctions ${ }^{5}$. The qualitative similarity is striking, and thus the present results seem to imply consistency with the model of Ref. 5 in which the effective coherent Josephson area, $A_{\text {eff, }}$ of the $\mathrm{Nb}$ junctions is experimentally shown to be $\Phi_{\mathrm{o}} / \mathrm{B}$ at high fields. Note that at low fields, A eff is limited by junction dimensions or defects to some fixed $A_{0}$; which we incorporate into the model by:

$$
A_{\text {eff }}=\Phi_{o} /\left(B+B_{o}\right)
$$

where $B_{o} \equiv \Phi_{o} / A_{o}$ : then the fits shown in Fig. 2 indicate $B_{o} \sim 0.05 T$, or $A_{o} \sim 0.04 \mu m^{2}$, for the $\mathrm{YBa}_{2} \mathrm{Cu}_{3} \mathrm{O}_{7-\delta}$ crystals.

In Fig. 3, we show experimental evidence of the extent to which it is the c-axis component of $B$ which primarily determines $R_{C}$ (as first described in Ref. 8 for $a b$ plane transport). The solid lines represent data for field sweeps with Bllc from three 
$Y \mathrm{Ba}_{2} \mathrm{Cu}_{3} \mathrm{O}_{7-\delta}$ crystals $\left(\mathrm{T}_{\mathrm{C}}=35,65\right.$ and $75 \mathrm{~K}$ ), while the symbols represent rotations at fixed fields (of 4,2 and $1.6 \mathrm{~T}$ ) for the same crystals. Note that for the $65 \mathrm{~K}$ crystal the rotation data for 4 and $2 \mathrm{~T}$ were taken at 51 and $49.5 \mathrm{~K}$ respectively, while the field sweep was done at $50 \mathrm{~K}$, leading to the slight systematic offsets shown. This new result is consistent with Josephson dissipation of Refs. 3-5 but not flux motion.

Encouraged by this excellent qualitative agreement with Josephson dissipation, we seek a more quantitative fit of the data using simple extensions of conventional Josephson models. To fit the data in Fig. 1, we use $\mathrm{e}^{10}$

$$
R_{c}(T)=R_{N c}(T)\left\{I_{0}\left(E_{j} / 2 k_{B} T\right)^{2}\right\}^{-1},
$$

which at low temperatures reduces to

$\dot{R}_{c}(\phi)=R_{N c} \exp \left(-\frac{e_{j}(T) \Phi_{o}}{\left(B \cos \phi+B_{0}\right) k_{B} T}\right)$

and is thus consistent with the Arrhenius activation leading to Fig. 2. Here the fitting constants are $R_{N c}$ and $e_{j}=E_{j} / A_{\text {eff }}$, since $B_{0}=0.05 T$ (see above). The excellent agreement with Eq. 3, shown in Fig. 1, is strong supporting evidence for the overall Josephson model of Refs. 3-5 including further confirmations of the 1/B dependence of $E_{j}$ and the significance of the c-axis component of $B$. We now express $e_{j}$ within the Anderson-Ambegaokar-Baratoff ${ }^{9}(\mathrm{AAB})$ model as:

$$
e_{j}(T)=\frac{\pi \hbar \Delta(T)}{2 e^{2} s \rho_{N c}} \tanh \left(\frac{\Delta(T)}{2 k_{B} T}\right)
$$

where $\mathrm{s}$ is the repeat distance of the $\mathrm{Cu}-\mathrm{O}$ bilayers, and ask whether values for the $\mathrm{c}$ axis resistivity, $\rho_{N c}$, and energy gap, $\Delta(T)$, can be found which are compatible with $R_{N c}$ and a suitable $\Delta(T)$. We expect $\rho_{N c}$ to be given by $R_{N c} A_{x t a l} / t_{x t a l}$, where $A_{x t a l}$ and $t_{x t a l}$ are the area and c-axis thickness of the crystal, but in that case there is a significant disagreement with a reasonable $\Delta(T)$ for rotational data taken at lower temperatures, and conversely, using $\Delta(T) / \Delta(0)$ from the BCS theory with $\Delta(0)=1$ to 1.4 times the $B C S$ value ${ }^{14}, \Delta_{B C S}(0)$, of $10 \mathrm{meV}$, there is a significant disagreement at lower temperatures with the expected $\rho_{\mathrm{Nc}}$.

In order to systematically address the discrepancy with the standard Josephson model ${ }^{9,10}$ we have measured $R_{c}$ for the crystal with $T_{C}=65 \mathrm{~K}$ over a wide range of $B$ and $T$ and include a parallel quasiparticle conductance in fits, as in Ref. 3. This 
procedure is more clearly presented in terms of the c-axis conductances, $Y_{C} \equiv 1 / R_{c}$, which are plotted in Fig. 4, and fit by 3,10

$$
\mathrm{Y}_{\mathrm{C}}(\mathrm{T})=\mathrm{Y}_{\mathrm{NC}}(\mathrm{T})\left\{\mathrm{I}_{\mathrm{o}}\left(\mathrm{E}_{\mathrm{j}} / 2 \mathrm{k}_{\mathrm{B}} \mathrm{T}\right)^{2}-1+\sigma_{\mathrm{qp}}(\mathrm{T})\right\}
$$

where the first two terms in brackets represent the pair conductance ${ }^{3}, I_{0}$ is the modified Bessel function ${ }^{10}$ and $\sigma_{\mathrm{qp}}(\mathrm{T})$ is the quasiparticle conductance, normalized to its normal-state value, which is given analytically in Taylor's thesis ${ }^{15}$. For the 60

- K data, the dotted curve represents only the pair conductance, while the solid curve includes $\sigma_{\mathrm{qp}}$, with $\Delta(\overline{0})=\Delta_{\mathrm{BCS}}(0)$. It is clear that $\sigma_{\mathrm{qp}}$ has little effect on the vast majority of the data which thus accurately determine $E_{j}$ and $Y_{\mathrm{Nc}}$ from Eq. 5.

As above, we cannot find values for $\rho_{\mathrm{Nc}}$ which are compatible with $Y_{\mathrm{Nc}}, E_{j}$ and a suitable $\Delta(\mathrm{T})$. To show this we plot in Fig. 5 the c-axis resistance of the $\mathrm{YBa}_{2} \mathrm{Cu}_{3} \mathrm{O}_{7-\delta}$ crystal $\left(T_{c}=65\right)$ in zero field as a function of $T$, together with values determined from the fits to Fig. 4 of $1 / Y_{N c}$ and $\rho_{N c} t_{x t a l} / A_{x t a l}$. Only the latter parameter is affected by $\Delta(0)$, through Eq. 4 , so they are shown for both $\Delta(0)=\Delta_{B C S}(0)$ and $141.4 \Delta_{B C S}(0)$. A similar problem occurs in fits ${ }^{3}$ to the c-axis resistivity of $\mathrm{Bi}_{2} \mathrm{Sr}_{2} \mathrm{CaCu}_{2} \mathrm{O}_{8}$ : using a temperature-independent $Y_{\mathrm{Nc}}$, the low-conductance fit to the Josephson model was consistent with the measured $Y_{N c}$ above $T_{c}$, but $Y(T)$ at low-temperatures needed a larger $\mathrm{E}_{\mathrm{j}}$.

A difficulty with this analysis is our inability to unambiguously determine the normal-state resistance, even above $T_{C}$, since expected fluctuation effects ${ }^{3}$ on $R_{N c}\left(T>T_{c}\right)$ cannot be easily estimated because the strictly two-dimensional nature of the $\mathrm{Cu}-\mathrm{O}$ bilayers is less certain in $\mathrm{YBa}_{2} \mathrm{Cu}_{3} \mathrm{O}_{7-\delta}$, with its partial chains, than for $\mathrm{Bi}_{2} \mathrm{Sr}_{2} \mathrm{CaCu}_{2} \mathrm{O}_{8}$. Nevertheless, we can speculate. One possibility is that the c-axis transport may be more coherent at low temperatures, either: below $T_{c}$, since the superconductivity will diminish in-plane fluctuations that would otherwise dephase adjacent bilayers ${ }^{16}$; or at low temperature such that the direct, metallic-like c-axis hopping rate exceeds that for boson assisted processes 17 . The smaller values and temperature dependence of $\rho_{N c} t_{x t a l} / A_{x t a l}$ compared to $R_{N c}\left(T \geq T_{C}\right)$ could result from this. For the opposite dependence of $1 / Y_{N c}$, it is possible that these $c$-axis Josephson junctions are not overdamped, and thus the prefactors for the pair conductance in Eqns. 2, 3 and 5 may not be simply the normal-state one. Data on extremely underdamped, discrete $\mathrm{Nb}-\mathrm{Pb}$ junctions find 18 a prefactor resistance which is inversely proportional to the quasiparticle density, increasing dramatically 
as $T$ decreases and equal to $R_{N}$ below $T_{c}$. The fit values of the prefactor $Y_{N c}$ could certainly be consistent with such a picture.

In conclusion, while our data undeniably shows that Josephson coupling and dissipation dominates for transport along the c-axis, the details indicate the possibility of an unusual normal-state conductance and that the c-axis Josephson junctions may be extremely underdamped. We also show for the first time that the $c$-axis component of magnetic field dominates the $c$-axis dissipation in most cases (except for fields nearly parallel to the ab planes).

We thank A. Leggett and K. Levin for helpful discussions. This research is supported by the U.S. Department of Energy, Basic Energy Sciences-Materials Sciences, and Energy Efficiency and Renewable Energy, as part of a program to develop electric power technology, under contract \#W-31-109-ENG-38, the National Science Foundation-Office of Science and Technology Centers under contract DMR 91-20000. ACF, WCJ and BRW supported by the Division of Educational Programs. [text word count $=1950$; characters $=11382$ ] 


\section{REFERENCES}

1. G. Briceno, M.F. Crommie and A. Zettl, Phys. Rev. Lett. 66, 2164 (1991).

2. R. Kleiner, F. Steinmeyer, G. Kunkel and P. Müller, Phys. Rev. Lett. 68, 2394 (1992); R. Kleiner and P. Müller, preprint.

3. K.E. Gray and D.H. Kim, Phys. Rev. Lett. 70, 1693 (1993).

4. D.H. Kim, K.E. Gray, R.T. Kampwirth, J.C. Smith, D.S. Richeson, T.J. Marks, J.H. Kang, J. Talvacchio and M. Eddy, Physica C177, 431 (1991).

5. D.H. Kim, K.E. Gray and J.H. Kang, Phys. Rev. B45, 7563 (1992).

6. K.E. Gray, D.H. Kim, B.W. Veal, G.T. Seidler, T.F. Rosenbaum and D.E. Farrell, Phys. Rev. B45, 10071 (1992).

7. K.E. Gray, R.T. Kampwirth, D.J. Miller, J.M. Murduck, D. Hampshire, R. Herzog and H.W. Weber, Physica C174, 340 (1991).

8. P.H. Kes, J. Aarts, J. van den Berg, C.J. van der Beek and J.A. Mydosh, Supercond. Sci. Technol. 1, 242 (1989).

9. P.W. Anderson, Lectures at Ravello Spring School, 1963; V. Ambegaokar and A. Baratoff, Phys. Rev. Lett. 10, 486 (1963).

10. V. Ambegaokar and B.I. Halperin, Phys. Rev. Lett. 22, 1364 (1969).

11. D. L. Kaiser, F. Holtzberg, M. F. Chisholm and T. K. Worthington, J. Crystal Growth 85, 593(1987).

12. J. D. Jorgensen, B. W. Veal, A. P. Paulikas, L. J. Nowicki, G. W. Crabtree, H. Claus and W. K. Kwok, Phys. Rev. B41, 1863 (1990).

13. A.F. Volkov and Y.I. Latyshev, JETP Lett. 53, 213 (1991).

14. Tunneling measurements consistently see features near $20 \mathrm{meV}$ for $\mathrm{YBa}_{2} \mathrm{Cu}_{3} \mathrm{O}_{7}$ with $\mathrm{T}_{\mathrm{c}} \sim 90 \mathrm{~K}$, i.e., roughly 1.4 times the $\mathrm{BCS}$ value, e.g., see $\mathrm{M}$. Kawasaki, et al, Jpn. J. Appl. Phys. 32, 1612 (1993). Such a large value is consistent with tunneling into many other HTS cuprates which range up to twice BCS, see J.F. Zasadzinski, et al, J. Phys. Chem. Solids 53, 1635 (1992).

15. B.N. Taylor, Thesis, University of Pennsylvania, 1963, unpublished, p. 50.

16. A.J. Leggett, private communication.

17. R.J. Radtke, C.N. Lau and K. Levin, preprint.

18. P. Silvestrini, O. Liengme and K.E. Gray, Phys. Rev. B37, 1525 (1988). 
Figure Captions

Fig. 1. The $c$-axis resistance of a $\mathrm{YBa}_{2} \mathrm{Cu}_{3} \mathrm{O}_{7-\delta}$ crystal $\left(\mathrm{T}_{\mathrm{C}}=65 \mathrm{~K}\right)$ as a function of the angle between the field direction and the c-axis taken at $60 \mathrm{~K}$ and $1 \mathrm{~T}$. The solid line is a fit to Eq. 3 for purely Josephson dissipation, using $R_{N c}=0.1$ ohms and $e_{j}(60 \mathrm{~K})=2.6 \times 10^{-6} \mathrm{~J} / \mathrm{m}^{2}$.

Fig. 2. The activation energy determined from Arrhenius plots of $R_{C}(T)$ as a function of $\mathrm{B}$ for $\phi=0$. Two $\mathrm{YBa}_{2} \mathrm{Cu}_{3} \mathrm{O}_{7-\delta}$ crystals $\left(\mathrm{T}_{\mathrm{c}}=65\right.$ and $\left.75 \mathrm{~K}\right)$ and two discrete, thin-film $\mathrm{Nb}$ junctions ${ }^{5}$ are shown (open symbols) to be strikingly similar with a 1/B dependence at high fields.

Fig. 3. The c-axis resistance of $\mathrm{YBa}_{2} \mathrm{Cu}_{3} \mathrm{O}_{7-\delta}$ crystals $\left(\mathrm{T}_{\mathrm{C}} \sim 35,65\right.$ and $\left.75 \mathrm{~K}\right)$ as a function of the c-axis component of $\mathrm{B}$. The solid lines are data for field sweeps (with $\phi=0$ ), while the symbols represent rotations at fixed fields (of 4 , 2 and $1.6 \mathrm{~T}$ ) for the same crystals. Note that for the $65 \mathrm{~K}$ crystal the data were taken at slightly different temperatures, which are consistent with the slight systematic offsets.

Fig. 4. The $c$-axis conductance of a $\mathrm{YBa}_{2} \mathrm{Cu}_{3} \mathrm{O}_{7-\delta}$ crystal $\left(\mathrm{T}_{\mathrm{c}}=65 \mathrm{~K}\right)$ for various temperatures, as a function of $B$ for $\phi=0$. Fits are shown to the pair conductance alone (dotted line for $60 \mathrm{~K}$ data only) and a sum of the pair and quasiparticle conductances (Eq. 5) for $\Delta(0)$ equal to the BCS value.

Fig. 5. The c-axis resistances of a $\mathrm{YBa}_{2} \mathrm{Cu}_{3} \mathrm{O}_{7-\delta}$ crystal $\left(\mathrm{T}_{\mathrm{c}}=65\right)$ in zero field as a function of $\mathrm{T}$ (open circles), together with values determined from the fits to Fig. 5 of $1 / Y_{\mathrm{Nc}}$ (triangles) and $\rho_{\mathrm{Nc}} \mathrm{t}_{\mathrm{xtal}} / \mathrm{A}_{\mathrm{xtal}}$. The latter are shown for $\Delta(0)$ equal to $\triangle_{\mathrm{BCS}}(0)$ (solid squares) and $1.4 \Delta_{\mathrm{BCS}}(0)$ (dashed line) since the values of $\rho_{N \mathrm{~N}}$, but not $E_{j}$, obtained from the fits in Fig. 5 depend on the actual (but unknown) magnitude of $\Delta(0)$. 


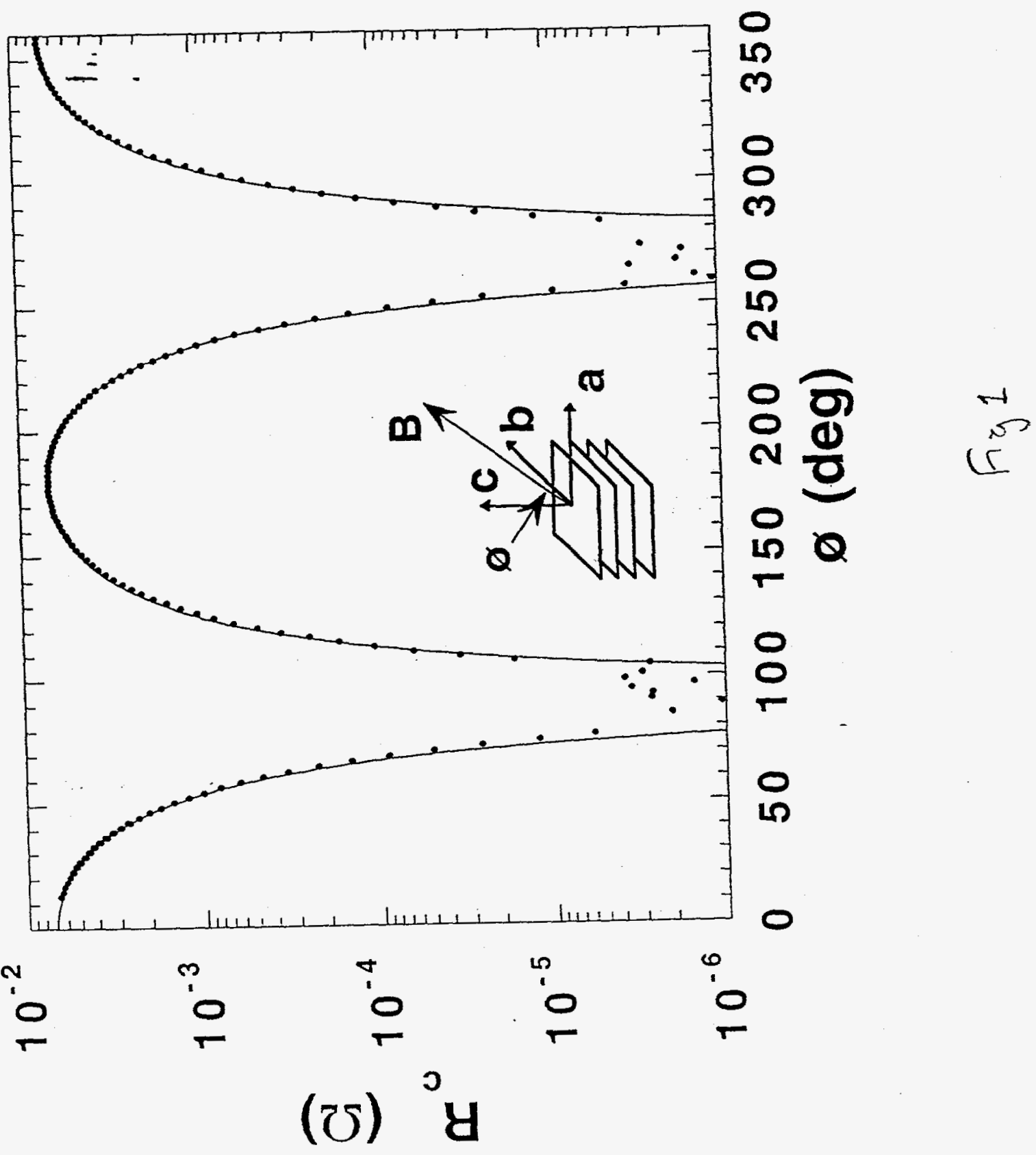




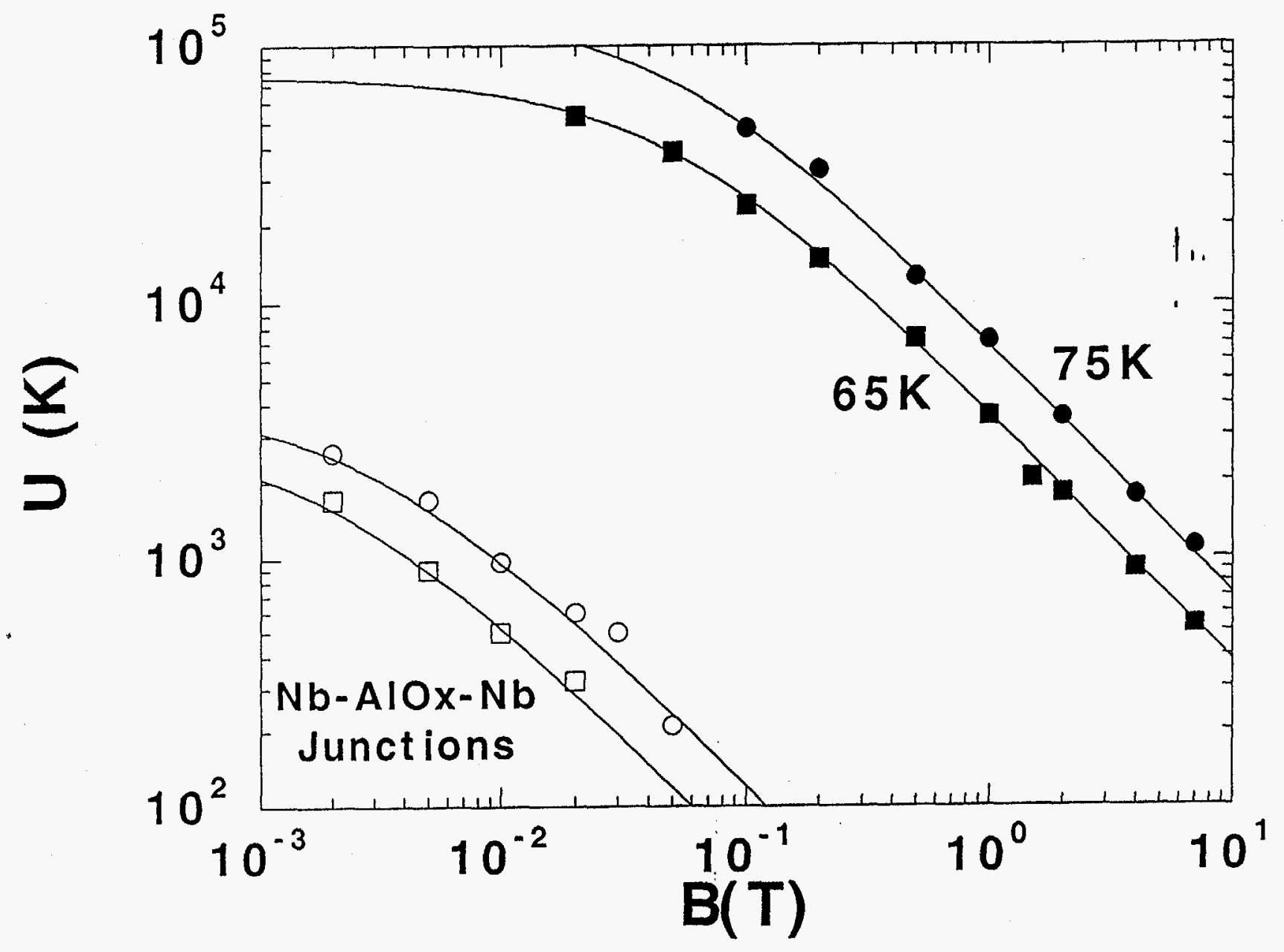

Fig 2 


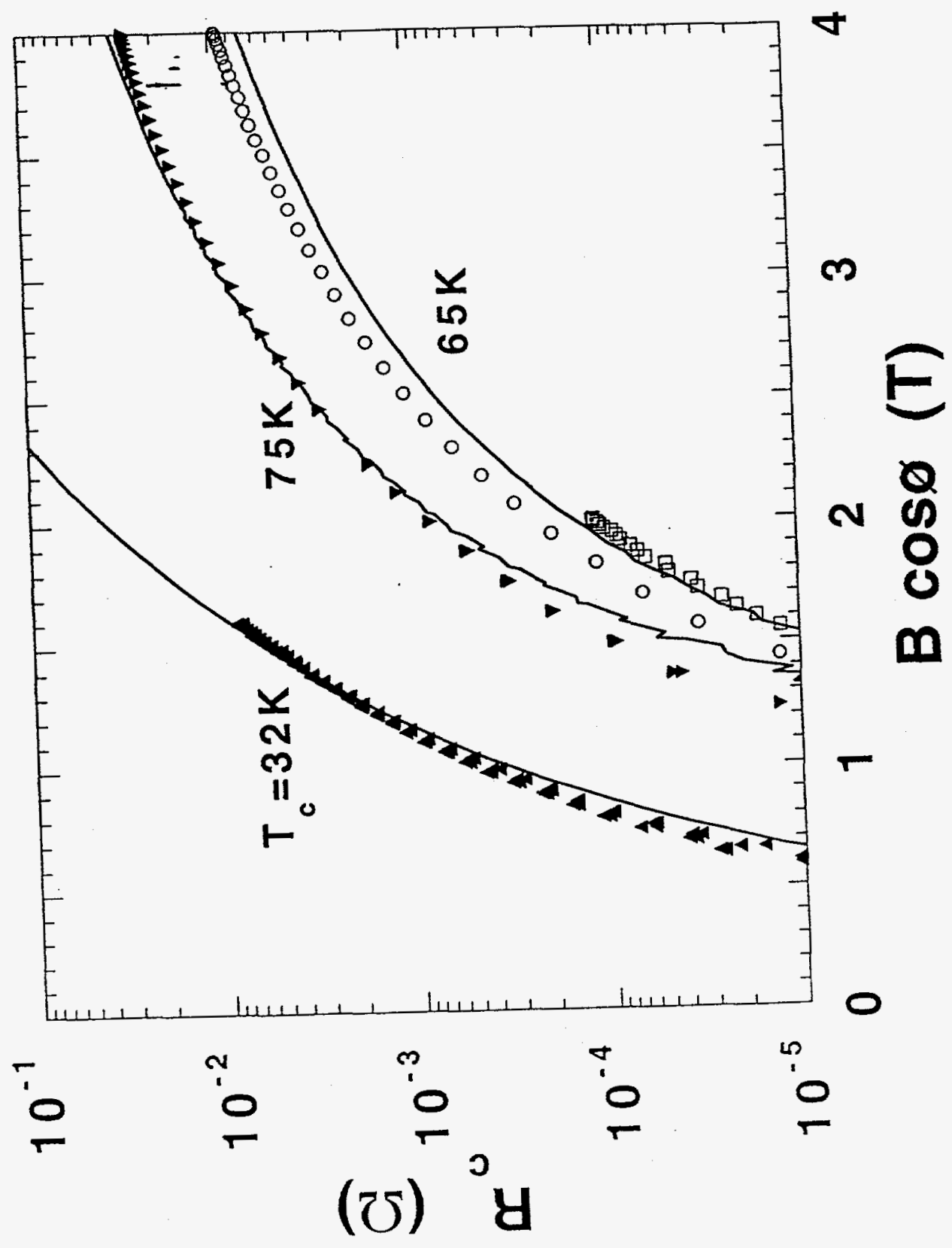

$m$ 1 


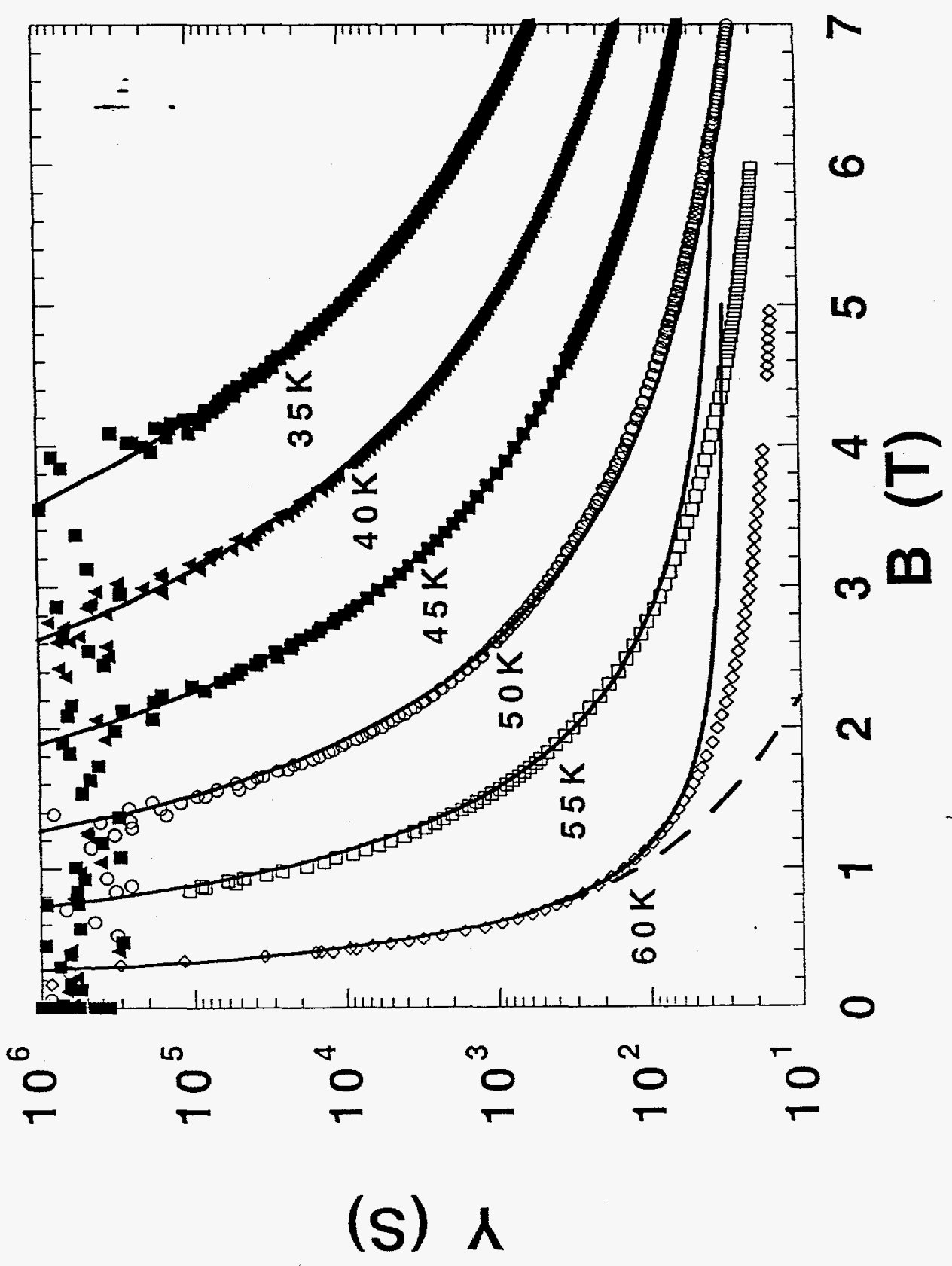

J

is 


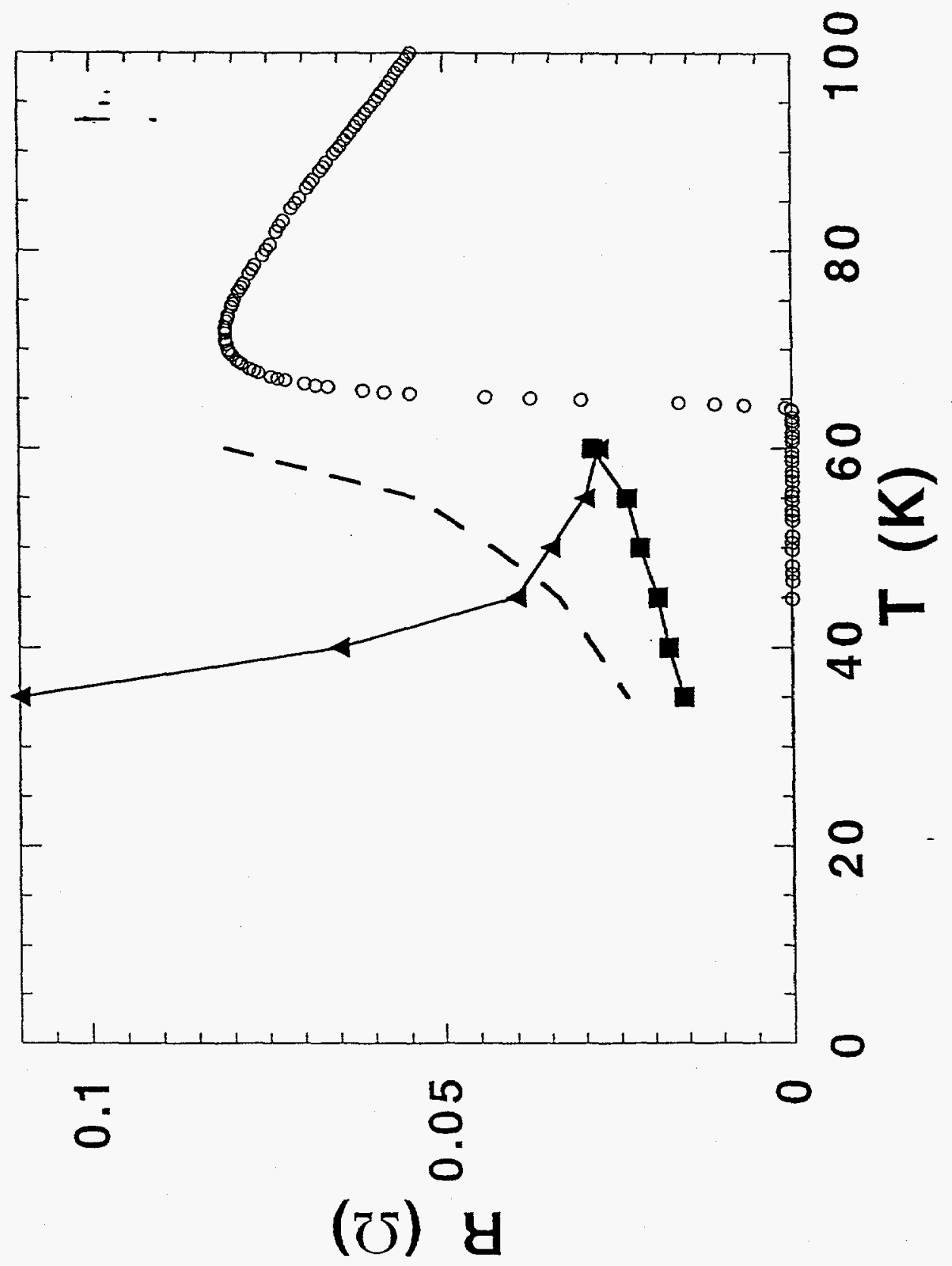

